\title{
A systematic literature review on synchronous hybrid learning: gaps identified \\ Annelies Raes ${ }^{1,2}$, Loulou Detienne ${ }^{1}$, Ine Windey ${ }^{1,2}$, Fien Depaepe ${ }^{1,2}$ \\ ${ }^{1}$ Faculty of Psychology and Educational Sciences, KU Leuven Kulak (Belgium), \\ Etienne Sabbelaan 53, 8500 Kortrijk. \\ ${ }^{2}$ ITEC, imec research group at KU Leuven (Belgium), Kapeldreef 75, 3001 Leuven.
}

Email: annelies.raes@kuleuven.be; loulou.detienne@telenet.be, ine.windey@kuleuven.be,

fien.depaepe@kuleuven.be,

\begin{abstract}
More and more higher educational institutions invest in technology-enhanced learning spaces, which raises the question of how these environments can be shaped to be as effective as possible. A specific new learning space is the synchronous hybrid or blended learning environment in which both face-to-face and remote students can simultaneously attend learning activities. Given synchronous blended learning is relatively new, there a few studies that have investigated its use and effectiveness. This study aims to synthesize the best available evidence worldwide to 1) have an overview of the state-of-the-art of the current research, to get a better insight in the do's and don'ts within this setting by summarizing 2) benefits, 3) challenges; and 4) to formulate design principles. Finally, 5) we also aimed to define the future directions for investigation based on the current research gaps. In line with the Preferred Reporting Items for Systematic Reviews and MetaAnalyses (PRISMA), this study included 47 studies which were analyzed to respond to our five research questions. One of the main findings is that existing research express cautious optimism about synchronous hybrid or blended learning which creates a more flexible, engaging learning environment compared to fully online or fully on-site instruction. Yet, this new learning environment has several challenges which are both pedagogical and technological in nature. To meet these challenges several design guidelines are formulated. A final conclusion is that most of the existing literature is still exploratory and qualitative in nature and has focused mostly on the description of students' experiences, the organizational implementation and the technological design. Empirical studies have only begun to emerge and more research is needed examining different pedagogical scenarios and its impact on student outcomes.
\end{abstract}

\section{Keywords}

Synchronous hybrid or blended learning; Here or There Instruction ; Systematic review; Research gaps

\section{Introduction}

Based on current societal transitions and in the context of the EU Lifelong Learning Program, both higher education and adult learning institutions are invited to constantly think about how to enable people, at any stage of their life, to take part in stimulating learning experiences. Regarding higher education settings, current policy documents often refer to the possibilities of multi-campus learning and inter-institutional collaboration by connecting remote groups with the traditional face-to-face classrooms (e.g., name omitted for blind review, 2018). Furthermore, the need for connecting remote individual students is increasing as the population in higher and adult education is getting more diverse. More and more "lifelong learners" cannot attend traditional classroom instruction due to, for example, family or work commitments. Within this context, digital technologies are often put forth as a possible answer to change the educational landscapes and make it more flexible and accessible for a larger group of learners (Cain, 2015). As access to synchronous communication tools improves, the lines between traditional face-to-face and online models of education (e.g. MOOCs) have become blurred, making way for new synchronous blended or hybrid approaches (Alexander, Lynch, Rabinovich \& Knutel, 2014; Roseth, Akcaoglu \& Zellner, 2013). Previous studies show that different models of synchromodal classes can be designed and implemented (Bell, Sawaya, \& Cain, 2014; Bower, Dalgarno, Kennedy, Lee \& Kenney, 2014, 2015).

Recently, at the university [institution and location omitted for blind review], two models of synchronous hybrid learning environments have been designed as displayed in Figure 1. 


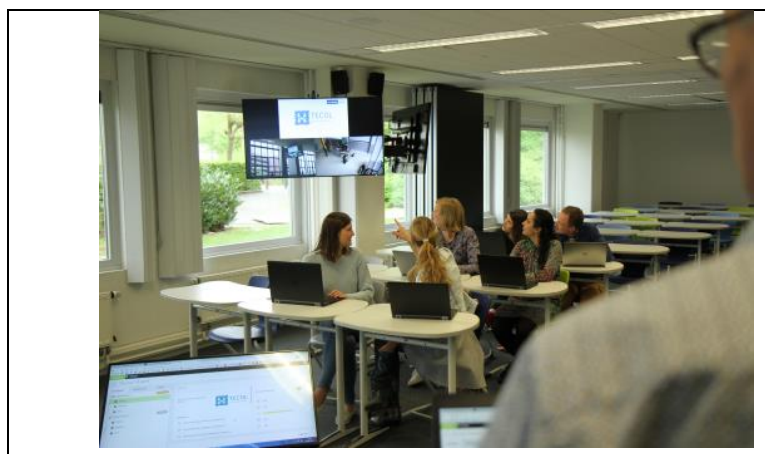

The Remote Classroom

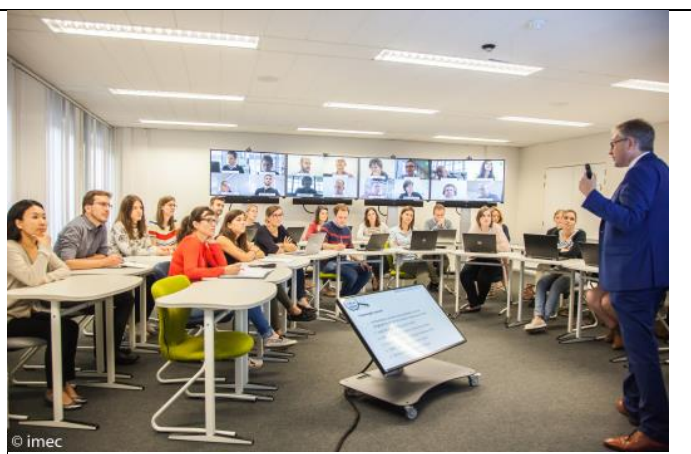

The Hybrid Virtual Classroom

Figure 1. Two models of synchronous hybrid learning at the living lab of the university

The picture on the left depicts what we call the "Remote Classroom", the picture on the right depicts the "Hybrid Virtual Classroom". Both learning settings have in common that both face-to face or "here" student and remote or "there" students are simultaneously included. This kind of learning and instruction is also framed as "Here or There (HOT) instruction (Zydney, McKimmy, Lindberg, \& Schmidt, 2019). The differences between the Remote and the Hybrid Virtual Classroom emerge from the location of which students follow the lecture. In the Remote Classroom setting, one group follows the course on campus and another group follows the course synchronously from another campus (the remote group is displayed on the screen depicted on the left corner of the picture in Figure 1) (Szeto \& Cheng, 2016). In the Hybrid Virtual Classroom, one group follows the course on campus and simultaneously individuals follow the course remotely from their location (Butz, Stupnisky, Pekrun, Jensen, \& Harsell, 2016; Hastie, Hung, Chen \& Kinshuk, 2010). This method of teaching offers even more flexibility because it gives adult students, as well as students who are, for example, abroad or ill for a longer period of time, the opportunity to participate in the actual lesson and to interact at a distance with all the students and the teacher from a place of their own choice. These learning environments have been constructed in collaboration with our industry partner [name omitted for blind review]. The newly designed learning spaces function as living labs to study new modes of teaching and learning. The two settings are equipped with innovative educational technology and all students have access to the same interactive platform shown in Figure 2, allowing them to participate in the course, either on-site or from a remote location. The platform gives access to the sources the teacher is using during his or her lecture (e.g. power point slides or annotations made on the digital whiteboard), facilitates launching quizzes or polls and is equipped with a chat room which gives students the possibility to chat with each other or with the teacher during the lecture. Lectures in the Hybrid Virtual Classroom are always assisted by a room controller who follows up on the chat, can launch the quiz or poll and can mute or unmute remote students. 


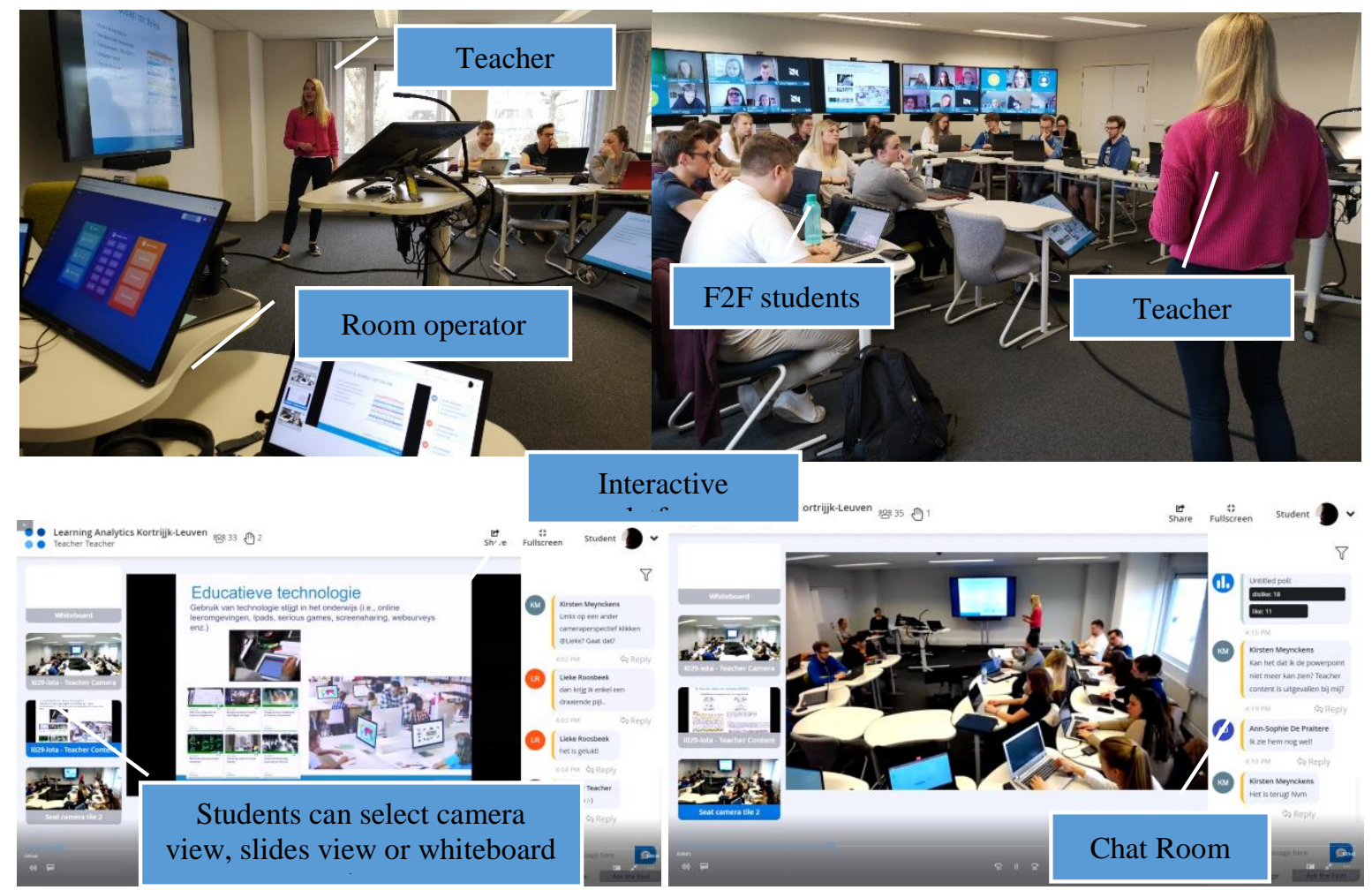

Figure 2. Upper pictures display the Hybrid Virtual Classroom including both F2F and remote individual students. Lower pictures display the platform visible for the students.

\section{Research objective}

We are at the start of the research project on synchronous hybrid learning and aimed to conduct a systematic review before starting new studies as the research field can learn a lot from earlier studies and prevents both the research field and practitioners from making the same mistakes. Without a systematic review, a new trial might add little to what is already known in the field (Baumeister \& Leary, 1997; Bettany-Saltikov, 2010a, 2010b).

In this case we aimed to summarize existing evidence concerning synchronous hybrid learning (e.g. benefits, challenges and design guidelines). Next to this, we aimed to identify existing gaps in current research in order to suggest areas for further investigation.

Our review questions are the following:

1. What is the state-of-the-art in research on synchronous hybrid learning?

2. What are the main benefits of synchronous hybrid learning?

3. What are the main challenges of synchronous hybrid learning settings?

4. What are the current design guidelines to optimize synchronous hybrid learning?

5. What are the research gaps in the current literature?

\section{Methodology}

\section{Inclusion and exclusion criteria}

As the setting under review is relatively new and one of the objectives was to find commonalities and gaps in research, the review considered studies that explored any aspect of synchronous hybrid learning and teaching. We did not predefine the population or the topic of interest the study should focus on. Neither, we predefined criteria related to the method, comparison or evolution of the study as we were especially interested in the kind of studies that have already been conducted. This means that a variety of quantitative and qualitative study designs were considered for inclusion. Also this review considered studies that explored any learner outcome (e.g. 
cognitive and affective outcomes) as long it was studied within the context of a synchronous hybrid or blended learning environment in the form of a remote classroom or a hybrid virtual classroom. This means that this review will not include literature focusing on the pure virtual classroom in which no face-to-face students were present.

\section{Search strategy}

A specific search strategy was followed to find both literature published in peer-reviewed journals and grey literature (including for example conference proceedings). This included a search of electronic databases and a manual search of the reference lists of all the identified relevant articles using the snowballing method. We systematically searched the following electronic databases: Web of Science, ERIC, Scopus, and LearnTechLib. Keyword descriptors for publications on synchronous hybrid learning and teaching comprised the following groups of search terms: (a) simultaneous, synchronous; (b) hybrid, hyflex, blended; (c) face-to-face, face to face; (d) education, teaching, learning. Search terms within each group were combined by means of a Boolean OR. The four groups of search terms were combined by means of a Boolean AND. In addition, to exclude studies on asynchronous learning this term was entered by means of Boolean NOT. Dependent on the options of the different databases, the results were further refined by the filters 'Education - educational research', 'Social Sciences', 'Peer reviewed only' and 'Education scientific disciplines'. This resulted in the following full search query:

$T S=($ simultaneous OR synchronous $)$ AND TS=(hyflex OR hybrid OR blended $)$ AND TS=(face-to-face OR face to face) $A N D T S=($ education OR teaching OR learning) NOT TS=(asynchronous)

Articles deemed relevant were retrieved for full-text review and assessed for inclusion using the pre-established selection criteria. Studies were limited to the English language. There were no date limitations placed on the review.

\section{Data analysis}

In order to obtain a systematic review of good quality the PRISMA guidelines (Preferred Reporting Items for Systematic Reviews and Meta-Analyses) were used. These guidelines consist of a checklist and a flow diagram, and help improve the reporting of the review. A summary of the search and selection process is presented in Figure 3 and is based on the PRISMA statement (Moher, Liberati, Tetzlaff, Altman \& The PRISMA Group, 2009). The Boolean search query in the four databases resulted in 286 studies, 92 were duplicated. This resulted in 194 studies which were screened based on the title and the abstracts. This identification and screening phase has been completed by the first two authors, independently form each other. It was checked in more detail whether the study involved a synchronous hybrid learning setting, yet in many cases this was not yet clear from the abstract and then these studies were selected for full text screening. In total 72 manuscripts remained for further assessment through reading the full text, this has been done by the first two authors. The results were discussed in weekly face-to-face meetings. Doubtful case were also screened by the third author. Many studies were removed $(n=36)$ as they did not meet the selection criteria. The most common reason for exclusion was the fact that the research was not about synchronous learning situations as stated above. For example, a study reporting about a blended course integrating Synchronous Online Discussion and Face-to-Face Instruction separately, but not combining this at the same time was removed (e.g. Blau et al., 2018) or a study reporting about synchronous learning but in a pure virtual class without face-to-face participants (e.g. Baker, \& Hjarlmarson, 2019). Based on the identification of the reference lists of the remaining studies, 11 additional studies were found. This resulted in 47 studies which were analyzed to answer the five research questions (see Table 2). In 7 cases, studies were clustered as the manuscript reported about the same learning setting and/or about the same set of participants. 


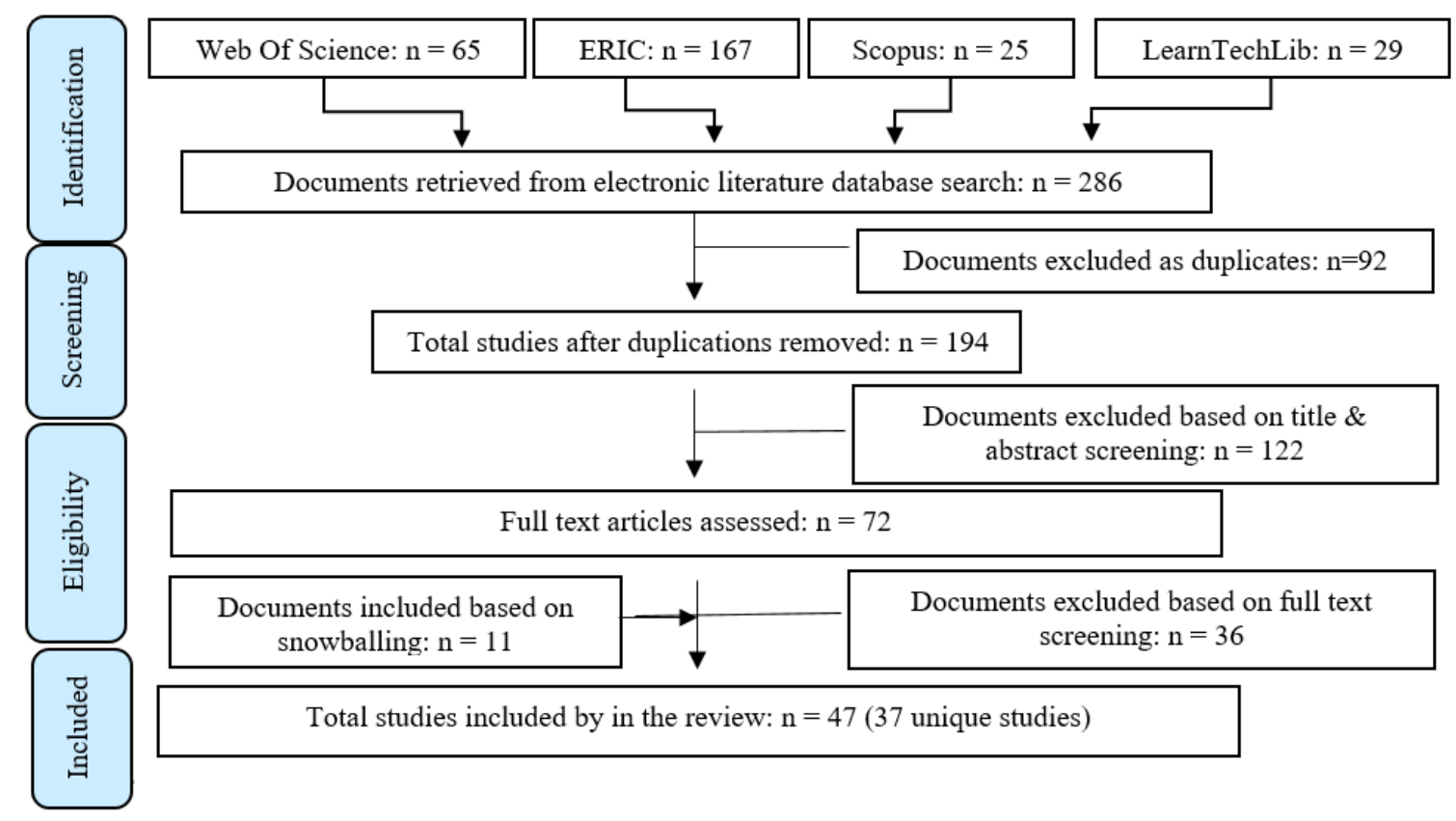

Figure 3. Overview of the search conducted in May 2019 based on PRISMA statement

\section{Results \\ RQ 1: Existing state-of-the-art research}

To answer Research Question 1, which aims to get insight in the SOTA, each publication was analyzed with regard to (a) the learning setting (Is the synchronous hybrid learning environment shaped as a Remote Classroom connecting groups or as a Hybrid Virtual Classroom connecting face-to-face participants with remote individuals?), (b) the context of the study and the number of participants, (c) the study design and research methodology, and (d) the study purpose. Table 2 summarizes the results of this analysis. The first study on synchronous hybrid learning date from 2003 , a qualitative case study aiming to observe the quantity and quality of human interaction between the instructor, the face-to-face students, and the distant students in a blended learning course. Also the work of Beatty $(2007,2010)$ was pioneering in the development, and evaluation of the HyFlex course design model for blended learning environments. The most studies date from a later period, i.e. published between 2013 and 2019. Most of the studies are case studies (28 in total), 15 of them using mixed methods, 13 of them only using qualitative analysis. Next, one review study and two conceptual studies were identified. Empirical studies are limited. Only five studies were found taken a comparative approach to study the effectiveness between different modes of delivery. Only one experimental study was found. This study was set up from a pretest-posttest experimental design with random assignment using a convergent parallel mixed methods approach (Butz \& Stupnisky, 2017). Almost all studies investigated the hybrid virtual classroom, namely 29. Only five studies report exclusively on the remote classroom, while three studies tackle both the remote as the hybrid virtual classroom in their study.. Lastly, almost all studies are conducted in the context of higher education or adult education settings, only one study focused on the pedagogical utilization of remote classrooms in contemporary elementary schools (i.e. Anastasiades et al., 2010). 
Table 2

Alphabetical overview of the included studies based on the systematic search

\begin{tabular}{|c|c|c|c|c|}
\hline & Study & $\begin{array}{l}\text { Learning setting, } \\
\text { context \& } \\
\text { participants }\end{array}$ & $\begin{array}{l}\text { Study design \& } \\
\text { methods }\end{array}$ & Study Purpose \\
\hline 1. & $\begin{array}{l}\text { Abdelmalak } \\
\& \text { Parra } \\
(2016)\end{array}$ & $\begin{array}{l}\text { Hybrid virtual } \\
\text { classroom } \\
\mathrm{N}=6 \text { graduate } \\
\text { students }\end{array}$ & $\begin{array}{l}\text { Qualitative case } \\
\text { study }\end{array}$ & $\begin{array}{l}\text { Exploration of students' perspectives } \\
\text { regarding HyFlex course design }\end{array}$ \\
\hline 2. & $\begin{array}{l}\text { Alexander, } \\
\text { Lynch, } \\
\text { Rabinovich } \\
\text { \& Knutel } \\
(2014)\end{array}$ & $\begin{array}{l}\text { Hybrid virtual } \\
\text { classroom } \\
\mathrm{N}=171 \text { university } \\
\text { students }\end{array}$ & $\begin{array}{l}\text { Mixed methods } \\
\text { case study }\end{array}$ & $\begin{array}{l}\text { Providing a snapshot of the hybrid } \\
\text { learning environment at Bentley } \\
\text { University that can be used as a model } \\
\text { by those in the planning stages or early } \\
\text { formation stages of a hybrid online } \\
\text { course or program and evaluation of } \\
\text { student's experiences }\end{array}$ \\
\hline 3. & $\begin{array}{l}\text { Anastasiades, } \\
\text { Filippousis, } \\
\text { Karvunis, } \\
\text { Siakas, } \\
\text { Tomazinakis, } \\
\text { Giza, \& } \\
\text { Mastoraki } \\
(2010)\end{array}$ & $\begin{array}{l}\text { Remote classroom } \\
\text { Context: K12 } \\
\text { education }- \text { grade } 6 \\
\mathrm{~N}=45 \text { students and } 4 \\
\text { teachers from } 2 \\
\text { schools }\end{array}$ & $\begin{array}{l}\text { Mixed methods } \\
\text { case study }\end{array}$ & $\begin{array}{l}\text { Presentation of the design, } \\
\text { implementation and evaluation of the } \\
\text { methodology which focuses on the } \\
\text { pedagogical utilization of Interactive } \\
\text { Videoconferencing (IVC) in } \\
\text { contemporary elementary schools. }\end{array}$ \\
\hline 4 & $\begin{array}{l}\text { Beatty }(2007, \\
2010)\end{array}$ & $\begin{array}{l}\text { Hybrid Virtual } \\
\text { classroom } \\
\text { Context: Adult and } \\
\text { higher education } \\
\mathrm{N}=34\end{array}$ & $\begin{array}{l}\text { Mixed methods } \\
\text { case study }\end{array}$ & $\begin{array}{l}\text { Description of the HyFlex course and } \\
\text { evaluation of student's participation and } \\
\text { satisfaction }\end{array}$ \\
\hline 5. & $\begin{array}{l}\text { Bell, Sawaya } \\
\text { \& Cain } \\
(2014)\end{array}$ & $\begin{array}{l}\text { Remote classroom \& } \\
\text { hybrid virtual } \\
\text { classroom } \\
\text { Context: Hybrid PhD } \\
\text { program } \\
\text { N not specified } \\
\end{array}$ & $\begin{array}{l}\text { Mixed method } \\
\text { case } \\
\text { study }\end{array}$ & $\begin{array}{l}\text { Description of different models of } \\
\text { synchromodal classes designed and } \\
\text { implemented }\end{array}$ \\
\hline 6. & $\begin{array}{l}\text { Bower, } \\
\text { Dalgarno, } \\
\text { Kennedy, } \\
\text { Lee, \& } \\
\text { Kenney } \\
(2014,2015) \\
\text { and Bower, } \\
\text { Lee, } \\
\text { Dalgarno } \\
(2017)\end{array}$ & $\begin{array}{l}\text { Remote classroom \& } \\
\text { hybrid virtual } \\
\text { classroom } \\
\text { Context: higher } \\
\text { education including } 7 \\
\text { design cases }\end{array}$ & $\begin{array}{l}\text { A cross-case } \\
\text { qualitative } \\
\text { analysis study }\end{array}$ & $\begin{array}{l}\text { To examine how design and } \\
\text { implementation factors influence student } \\
\text { learning activity and perceived learning } \\
\text { outcomes and describe this in a Blended } \\
\text { Synchronous Learning Design } \\
\text { Framework. }\end{array}$ \\
\hline 7. & $\begin{array}{l}\text { Brumfield, } \\
\text { Carleo, } \\
\text { Kenny, } \\
\text { Melendez, } \\
\text { O’Neill, } \\
\text { Polanin, \& } \\
\text { Reynolds- } \\
\text { Allie (2017) }\end{array}$ & $\begin{array}{l}\text { Remote classroom } \\
\text { Context: adult } \\
\text { education }\end{array}$ & $\begin{array}{l}\text { Qualitative case } \\
\text { study }\end{array}$ & $\begin{array}{l}\text { Description of the concept and the } \\
\text { design of the course }\end{array}$ \\
\hline 8. & $\begin{array}{l}\text { Butz \& } \\
\text { Askim- } \\
\text { Lovseth } \\
(2015)\end{array}$ & $\begin{array}{l}\text { Hybrid virtual } \\
\text { classroom } \\
\text { Context: higher } \\
\text { education }\end{array}$ & $\begin{array}{l}\text { Exploratory } \\
\text { quantitative study } \\
\text { comparing } \\
\text { different student }\end{array}$ & $\begin{array}{l}\text { Examination of the relationships among } \\
\text { attendance mode, student nationality and } \\
\text { oral communication assessment scores in } \\
\text { a synchronous hybrid programme. }\end{array}$ \\
\hline
\end{tabular}




\begin{tabular}{|c|c|c|c|c|}
\hline & & $\begin{array}{l}\mathrm{N}=202 \text { graduate } \\
\text { students, } 120 \text { on- } \\
\text { campus and } 82 \text { online }\end{array}$ & $\begin{array}{l}\text { groups: online vs. } \\
\text { on-campus, and } \\
\text { domestic vs. } \\
\text { international }\end{array}$ & \\
\hline 9. & $\begin{array}{l}\text { Butz \& } \\
\text { Stupnisky } \\
(2016,2017)\end{array}$ & 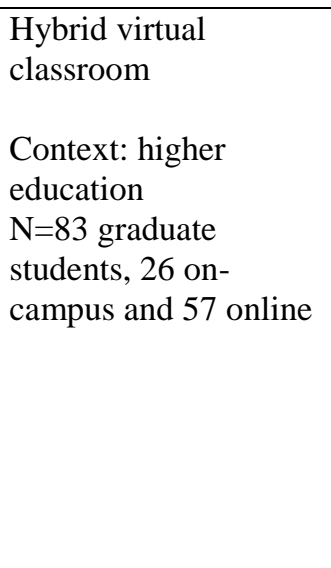 & $\begin{array}{l}\text { Pretest-posttest } \\
\text { experimental } \\
\text { design with } \\
\text { random } \\
\text { assignment to } \\
\text { either the } \\
\text { experimental } \\
\text { group or the } \\
\text { control group, } \\
\text { using a } \\
\text { convergent } \\
\text { parallel mixed } \\
\text { methods } \\
\text { approach. }\end{array}$ & $\begin{array}{l}\text { Implementation and evaluation of an } \\
\text { online discussion board intervention } \\
\text { designed to scaffold feelings of } \\
\text { relatedness and self-efficacy in } \\
\text { synchronous hybrid learning. }\end{array}$ \\
\hline 10. & $\begin{array}{l}\text { Butz, } \\
\text { Stupnisky, } \\
\text { Pekrun, } \\
\text { Jensen, \& } \\
\text { Harsell, } \\
\text { (2016) }\end{array}$ & $\begin{array}{l}\text { Hybrid virtual } \\
\text { classroom } \\
\text { Context: higher } \\
\text { education } \\
\text { N Time } 1=118 \\
\text { students, } 48 \text { on- } \\
\text { campus and } 70 \text { online } \\
\text { N Time } 2=100 \\
\text { students, } 37 \text { on- } \\
\text { campus and } 63 \text { online }\end{array}$ & $\begin{array}{l}\text { Exploratory } \\
\text { quantitative study } \\
\text { comparing online } \\
\text { vs. on-campus } \\
\text { students using } \\
\text { longitudinal } \\
\text { analyses. }\end{array}$ & $\begin{array}{l}\text { To investigate students' self-reported } \\
\text { enjoyment, anxiety, and boredom as } \\
\text { predictors of their program achievement } \\
\text { and successful technology use. }\end{array}$ \\
\hline 11. & Cain (2015) & $\begin{array}{l}\text { Hybrid virtual } \\
\text { classroom } \\
\text { Context: higher } \\
\text { education } \\
\text { N not specified }\end{array}$ & $\begin{array}{l}\text { Qualitative case } \\
\text { study }\end{array}$ & $\begin{array}{l}\text { Description of how instructors and } \\
\text { support staff involved in the hybrid } \\
\text { progra and explanation of their } \\
\text { innovative solution, i.e. the role of an in- } \\
\text { class technology navigator. }\end{array}$ \\
\hline 12. & $\begin{array}{l}\text { Cain, Bell \& } \\
\text { Cheng } \\
(2016)\end{array}$ & $\begin{array}{l}\text { Hybrid virtual } \\
\text { classroom } \\
\text { Context: } \mathrm{PhD} \\
\text { program } \\
\mathrm{N}=12 \text { doctoral } \\
\text { students (11 remote, } 1 \\
\text { F2F), } 1 \text { instructor, } 1 \\
\text { teaching assistant } \\
\text { (TA), and } 1 \text { TechNav. }\end{array}$ & $\begin{array}{l}\text { Qualitative case } \\
\text { study with focus } \\
\text { on the design and } \\
\text { use of the one } \\
\text { specific } \\
\text { application }\end{array}$ & $\begin{array}{l}\text { Evaluation of the robotic telepresence } \\
\text { devices to bring greater individualization } \\
\text { to online students in one particular } \\
\text { synchronous hybrid course. }\end{array}$ \\
\hline 13. & $\begin{array}{l}\text { Cunningham } \\
\text { (2014) }\end{array}$ & $\begin{array}{l}\text { Hybrid virtual } \\
\text { classroom } \\
\text { Context: postgraduate } \\
\text { education } \\
\mathrm{N}=4 \text { students } \\
\text { followed during real- } \\
\text { time online } \\
\text { participation }\end{array}$ & $\begin{array}{l}\text { Qualitative case } \\
\text { study }\end{array}$ & $\begin{array}{l}\text { Evaluation of the experiences of both } \\
\text { online and campus students in light of } \\
\text { social presence and activity theory }\end{array}$ \\
\hline 14. & $\begin{array}{l}\text { Grant \& } \\
\text { Cheon } \\
(2007)\end{array}$ & $\begin{array}{l}\text { Hybrid virtual } \\
\text { classroom } \\
\text { Context: higher } \\
\text { education }\end{array}$ & $\begin{array}{l}\text { Mixed method } \\
\text { effectiveness } \\
\text { study comparing } \\
\text { video and audio }\end{array}$ & $\begin{array}{l}\text { Research on how synchronous } \\
\text { conferencing technology affects teaching } \\
\text { and learning. and exploration of the } \\
\text { factors bearing on the success and failure }\end{array}$ \\
\hline
\end{tabular}




\begin{tabular}{|c|c|c|c|c|}
\hline & & $\begin{array}{l}\mathrm{N}=18, \text { One group } \\
\text { used video } \\
\text { conferencing } \\
\text { exclusively }(\mathrm{n}=11) \\
\text { and the other group } \\
\text { used only audio } \\
\text { conferencing }(\mathrm{n}=8)\end{array}$ & $\begin{array}{l}\text { conferencing in } \\
\text { hybrid classes. }\end{array}$ & $\begin{array}{l}\text { of synchronous conferencing in hybrid } \\
\text { classes. }\end{array}$ \\
\hline 15. & $\begin{array}{l}\text { Hastie, } \\
\text { Hung, Chen } \\
\& \text { Kinshuk } \\
(2010)\end{array}$ & $\begin{array}{l}\text { Hybrid virtual } \\
\text { classroom } \\
\text { Context: international } \\
\text { collaboration between } \\
\text { two educators } \\
\text { involving two } \\
\text { institutions from two } \\
\text { countries in the Asia- } \\
\text { Pacific region }\end{array}$ & $\begin{array}{l}\text { Description of } 9 \\
\text { design modes and } \\
\text { empirical case } \\
\text { study } \\
\text { Data collected } \\
\text { over 5-year period }\end{array}$ & $\begin{array}{l}\text { Description of nine modes of } \\
\text { synchronous hybrid learning and } \\
\text { investigation of the educational and } \\
\text { social gains }\end{array}$ \\
\hline 16. & $\begin{array}{l}\text { Huang, Shu, } \\
\text { Zhao, \& } \\
\text { Huang } \\
\text { (2017) }\end{array}$ & $\begin{array}{l}\text { Hybrid virtual } \\
\text { classroom } \\
\text { Context: } 5 \text { teachers } \\
\text { and students from } 2 \\
\text { senior schools in } \\
\text { china (N not specified } \\
\text { ) }\end{array}$ & $\begin{array}{l}\text { Mixed methods } \\
\text { case study }\end{array}$ & $\begin{array}{l}\text { Study on (1) how the teachers' activities } \\
\text { impact teaching effect } \\
\text { in their courses? (2) What do high school } \\
\text { students expect of their video teachers? } \\
\text { (3) What actions do remote students take } \\
\text { to achieve good learning experience? }\end{array}$ \\
\hline 17. & $\begin{array}{l}\text { Lakhal, } \\
\text { Bateman \& } \\
\text { Bédard } \\
(2017) \\
\end{array}$ & $\begin{array}{l}\text { Hybrid virtual } \\
\text { classroom } \\
\text { Context: Higher } \\
\text { education }\end{array}$ & Review study & $\begin{array}{l}\text { Description of the advantages, } \\
\text { challenges, conditions of success and the } \\
\text { formulation of a blended session } \\
\text { protocol. }\end{array}$ \\
\hline 18. & $\begin{array}{l}\text { Lightner \& } \\
\text { Lightner- } \\
\text { Laws (2016) }\end{array}$ & $\begin{array}{l}\text { Remote classroom } \\
\text { Context: higher } \\
\text { education } \\
\text { Analysis of data } \\
\text { collected from all } \\
\text { courses offered fall } \\
2009 \text { to fall } 2011 .\end{array}$ & $\begin{array}{l}\text { Empirical study } \\
\text { comparing course } \\
\text { delivery modes: } \\
\text { online, remote } \\
\text { and traditional } \\
\text { and its impact on } \\
\text { students grades } \\
\text { In the timeframe } \\
\text { of } 3 \text { years, there } \\
\text { were } 112,973 \\
\text { grades issued } \\
\text { across } 6316 \\
\text { courses. }\end{array}$ & $\begin{array}{l}\text { Impact of the environment on student } \\
\text { performance }\end{array}$ \\
\hline 19. & $\begin{array}{l}\text { Liu, Spector, } \\
\text { \& Ikle (2018) }\end{array}$ & $\begin{array}{l}\text { Hybrid virtual } \\
\text { classroom } \\
\text { Context: } 4 \\
\text { universities were } \\
\text { included taking turns } \\
\text { in designing, } \\
\text { developing and } \\
\text { delivering courses in } \\
\text { Computational } \\
\text { Science and } \\
\text { Engineering. }\end{array}$ & $\begin{array}{l}\text { Case study from a } \\
\text { developmental } \\
\text { approach }\end{array}$ & $\begin{array}{l}\text { Sharing the finding of the project } \\
\text { focusing on computer technologies } \\
\text { served as the enabler for course } \\
\text { development, student projects for model- } \\
\text { based learning, and course delivery } \\
\text { across different locations. }\end{array}$ \\
\hline 20. & $\begin{array}{l}\text { McGovern \& } \\
\text { Barnes } \\
(2009)\end{array}$ & $\begin{array}{l}\text { Hybrid virtual } \\
\text { classroom } \\
\text { Context: postgraduate } \\
\text { degree program in }\end{array}$ & $\begin{array}{l}\text { Mixed methods } \\
\text { case study }\end{array}$ & $\begin{array}{l}\text { Examination of why students choose to } \\
\text { participate virtually and the impact of } \\
\text { the } \\
\text { virtual classroom on learning and } \\
\text { communication. }\end{array}$ \\
\hline
\end{tabular}




\begin{tabular}{|c|c|c|c|c|}
\hline & & $\begin{array}{l}\text { advanced clinical } \\
\text { pediatrics } \\
\mathrm{N}=16 \text { students }\end{array}$ & & \\
\hline 21. & $\begin{array}{l}\text { Nortvig } \\
(2013)\end{array}$ & $\begin{array}{l}\text { Hybrid virtual } \\
\text { classroom } \\
\text { Context: professional } \\
\text { Bachelor program in } \\
\text { physiotherapy in } \\
\text { Denmark }\end{array}$ & $\begin{array}{l}\text { Conceptual study } \\
\text { focusing on } \\
\text { technological } \\
\text { design }\end{array}$ & $\begin{array}{l}\text { Investigation and description of how } \\
\text { technology can affect teaching in the } \\
\text { synchronous hybrid classroom } \\
\text { Explaining the concept of embodiment } \\
\text { of technology, technological } \\
\text { transformation and the influence of } \\
\text { technology. }\end{array}$ \\
\hline 22. & Olt (2018) & $\begin{array}{l}\text { Hybrid virtual } \\
\text { classroom } \\
\mathrm{N}=9 \text { remote students }\end{array}$ & $\begin{array}{l}\text { Qualitative case } \\
\text { study using } \\
\text { phenomenological } \\
\text { methodology }\end{array}$ & $\begin{array}{l}\text { To investigate the phenomenon of using } \\
\text { synchronous online classes blended with } \\
\text { a face-to-face classroom from the } \\
\text { perspective of the remote participant. } \\
\text { The study is situated within the initiative } \\
\text { "Bridge to Campus" providing the entire } \\
\text { freshman year of college through } \\
\text { synchronous online coursework. }\end{array}$ \\
\hline 23. & $\begin{array}{l}\text { Ørngreen, } \\
\text { Levinsen, } \\
\text { Jelsbak, } \\
\text { Moller, \& } \\
\text { Bendsen } \\
(2015)\end{array}$ & $\begin{array}{l}\text { Hybrid virtual } \\
\text { classroom } \\
\text { Context: The } \\
\text { Bachelor Programme } \\
\text { in Biomedical } \\
\text { Laboratory Analysis } \\
\text { in Aarhus }\end{array}$ & $\begin{array}{l}\text { Qualitative case } \\
\text { study as a } \\
\text { participatory } \\
\text { action research } \\
\text { project }\end{array}$ & $\begin{array}{l}\text { To identify potentials and barriers from } \\
\text { an ICT-supported learning perspective; } \\
\text { to develop robust educational designs } \\
\text { and teaching scenarios, and to qualify } \\
\text { teaching staff in teaching activities } \\
\text { which involve the use of the blended } \\
\text { class model }\end{array}$ \\
\hline 24. & $\begin{array}{l}\text { Ramsey, } \\
\text { Evans \& } \\
\text { Levy (2016) }\end{array}$ & $\begin{array}{l}\begin{array}{l}\text { Hybrid virtual } \\
\text { classroom }\end{array} \\
\text { Context: Public } \\
\text { university } \\
\mathrm{N}=19\end{array}$ & $\begin{array}{l}\text { Mixed methods } \\
\text { case study }\end{array}$ & $\begin{array}{l}\text { To present preliminary reflections on } \\
\text { their initial experiences and present their } \\
\text { survey data regarding students' } \\
\text { experiences }\end{array}$ \\
\hline 25. & $\begin{array}{l}\text { Rasmussen } \\
(2003)\end{array}$ & $\begin{array}{l}\begin{array}{l}\text { Hybrid virtual } \\
\text { classroom }\end{array} \\
\mathrm{N}=6 \text { remote students } \\
\text { living in various parts } \\
\text { of the western United } \\
\text { States }+11 \text { face-to- } \\
\text { face students on } \\
\text { campus }\end{array}$ & $\begin{array}{l}\text { Qualitative case } \\
\text { study }\end{array}$ & $\begin{array}{l}\text { To observe the quantity and quality of } \\
\text { human interaction between the } \\
\text { instructor, the face-to-face students, and } \\
\text { the distant students in a blended learning } \\
\text { course. }\end{array}$ \\
\hline 26. & $\begin{array}{l}\text { Romero-Hall } \\
\text { \& Vicentini } \\
(2017)\end{array}$ & $\begin{array}{l}\text { Hybrid virtual } \\
\text { classroom, } \\
\text { Context: graduate } \\
\text { level } \\
\begin{array}{l}\mathrm{N}=3 \text { graduate } \\
\text { students }\end{array}\end{array}$ & $\begin{array}{l}\text { Qualitative case } \\
\text { study }\end{array}$ & $\begin{array}{l}\text { To help inform the design of hybrid } \\
\text { synchronous instruction and to } \\
\text { understand the effectiveness and } \\
\text { efficiency of hybrid synchronous } \\
\text { instruction from the perspective of the } \\
\text { distance learners }\end{array}$ \\
\hline 27. & $\begin{array}{l}\text { Roseth, } \\
\text { Akcaoglu \& } \\
\text { Zellner } \\
\text { (2013) }\end{array}$ & $\begin{array}{l}\text { Hybrid virtual } \\
\text { classroom } \\
\text { Context: Hybrid } \\
\text { Doctoral Seminar }\end{array}$ & Conceptual study & $\begin{array}{l}\text { Description of the rationale behind } \\
\text { pedagogical choices and specification } \\
\text { how various technologies were re- } \\
\text { purposed to create a virtual classroom }\end{array}$ \\
\hline
\end{tabular}




\begin{tabular}{|c|c|c|c|c|}
\hline 28 . & $\begin{array}{l}\text { Shen, Wang, } \\
\& \text { Pan } \\
(2008)\end{array}$ & $\begin{array}{l}\text { Remote classroom } \\
\mathrm{N}=1000 \text { students, } \\
250 \text { on campus and } \\
750 \text { online }\end{array}$ & $\begin{array}{l}\text { Mixed methods } \\
\text { case study }\end{array}$ & $\begin{array}{l}\text { Technical description of the self- } \\
\text { developed interactive MLearning system } \\
\text { and evaluation }\end{array}$ \\
\hline 29. & $\begin{array}{l}\text { Stewart, } \\
\text { Harlow, \& } \\
\text { DeBacco } \\
(2011)\end{array}$ & $\begin{array}{l}\text { Hybrid virtual } \\
\text { classroom } \\
\mathrm{N}=46 \text { graduate } \\
\text { students were enrolled } \\
\text { in different courses } \\
\text { held over the two year } \\
\text { project }\end{array}$ & $\begin{array}{l}\text { Mixed methods } \\
\text { ethnographic } \\
\text { study }\end{array}$ & $\begin{array}{l}\text { Studying the experiences of learners } \\
\text { participating in multi-site education } \\
\text { classes }\end{array}$ \\
\hline 30. & $\begin{array}{l}\text { Szeto \& } \\
\text { Cheng } \\
(2016) \text { and } \\
\text { Szeto (2014, } \\
\text { 2015) }\end{array}$ & $\begin{array}{l}\text { Remote classroom } \\
\text { Context: computer- } \\
\text { aided engineering } \\
\text { drawing course } \\
\mathrm{N}=28 \text { students, } 14 \\
\text { face-to-face, } 14 \text { as } \\
\text { remote group }\end{array}$ & $\begin{array}{l}\text { Qualitative case } \\
\text { study }\end{array}$ & $\begin{array}{l}\text { Impact of the environment on students' } \\
\text { social presence experience }\end{array}$ \\
\hline 31. & $\begin{array}{l}\text { Vu \& Fadde } \\
(2013)\end{array}$ & $\begin{array}{l}\text { Hybrid virtual } \\
\text { classroom } \\
\text { Context: Two sections } \\
\text { of a graduate level } \\
\text { Multimedia Design } \\
\text { course were analyzed: } \\
\text { semester } 1: \mathrm{N}=15 \text {, } \\
\text { semester } 2, \mathrm{~N}=13\end{array}$ & $\begin{array}{l}\text { Mixed methods } \\
\text { case study }\end{array}$ & $\begin{array}{l}\text { To explore 1) students' choices of verbal } \\
\text { and text interaction and 2) explore } \\
\text { students' preference for online or remote } \\
\text { when given the choice. }\end{array}$ \\
\hline 32. & $\begin{array}{l}\text { Wang, Quek, } \\
\text { Hu (2017), } \\
\text { Wang, } \\
\text { Huang, \& } \\
\text { Quek (2018), } \\
\text { \& Wang \& } \\
\text { Huang } \\
(2018)\end{array}$ & $\begin{array}{l}\begin{array}{l}\text { Hybrid virtual } \\
\text { classroom }\end{array} \\
\mathrm{N}=24 \text { graduate } \\
\text { students (in-service } \\
\text { school teachers) } \\
\text { during } 13 \text { sessions of } \\
3 \text { hours }\end{array}$ & $\begin{array}{l}\text { Design based } \\
\text { research } \\
\text { (preliminary } \\
\text { research, } \\
\text { prototyping and } \\
\text { assessment) }\end{array}$ & $\begin{array}{l}\text { Description of benefits, challenges \& } \\
\text { providing pedagogical, social and } \\
\text { technical design principles of a blended } \\
\text { synchronous learning environment. }\end{array}$ \\
\hline 33. & $\begin{array}{l}\text { Weitze } \\
(2015) \& \\
\text { Weitze, } \\
\text { Ørngreen \& } \\
\text { Levinsen } \\
(2013)\end{array}$ & $\begin{array}{l}\text { Hybrid virtual } \\
\text { classroom } \\
\text { Context: adult } \\
\text { learning } \\
2 \text { classes included, } \\
\mathrm{N}=10+\mathrm{N}=26 \\
\end{array}$ & $\begin{array}{l}\text { Mixed methods } \\
\text { case study with } \\
\text { focus on design } \\
\text { perspective }\end{array}$ & $\begin{array}{l}\text { Description of students' experiences, the } \\
\text { organizational implementation and the } \\
\text { development of instructional design, the } \\
\text { IT-Pedagogical Think Tank for Teacher } \\
\text { Teams }\end{array}$ \\
\hline 34. & $\begin{array}{l}\text { White, } \\
\text { Ramirez, } \\
\text { Smith, \& } \\
\text { Plonowski } \\
\text { (2010) }\end{array}$ & $\begin{array}{l}\text { Hybrid virtual } \\
\text { classroom } \\
\text { Context: higher } \\
\text { education } \\
\mathrm{N}=10 \text { participants }\end{array}$ & $\begin{array}{l}\text { Mixed methods } \\
\text { case study }\end{array}$ & $\begin{array}{l}\text { Determination of the feasibility of } \\
\text { delivering a course } \\
\text { on-campus and in real time, } \\
\text { simultaneously } \\
\text { transmitting it to students who were } \\
\text { remotely } \\
\text { accessing the same course. }\end{array}$ \\
\hline 35. & $\begin{array}{l}\text { Wiles \& Ball } \\
(2013)\end{array}$ & 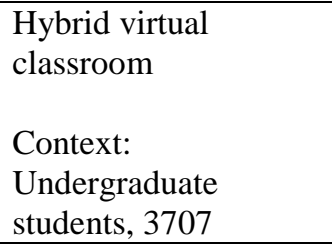 & $\begin{array}{l}\text { Longitudinal } \\
\text { mixed methods } \\
\text { case study }\end{array}$ & $\begin{array}{l}\text { Design of the converged classroom and } \\
\text { the description of benefits and } \\
\text { challenges }\end{array}$ \\
\hline
\end{tabular}




\begin{tabular}{|c|c|c|c|c|}
\hline & & $\begin{array}{l}\text { enrollments over } 7 \\
\text { semesters }\end{array}$ & & \\
\hline 36. & $\begin{array}{l}\text { Yen \& } \\
\text { Abdous } \\
\text { (2012) }\end{array}$ & $\begin{array}{l}\text { Combination of } \\
\text { Hybrid virtual and } \\
\text { remote classroom } \\
\mathrm{N}=496 \text { university } \\
\text { students }\end{array}$ & Empirical study & $\begin{array}{l}\text { Exploration of the relationships between } \\
\text { self-perceived learner-to-teacher } \\
\text { interaction and learning outcomes and } \\
\text { satisfaction across various learning } \\
\text { delivery modes (F2F, Satellite broadcast } \\
\text { or live video-streaming) }\end{array}$ \\
\hline 37. & $\begin{array}{l}\text { Zydney, } \\
\text { McKimmy, } \\
\text { Lindberg, \& } \\
\text { Schmidt, } \\
\text { (2019); } \\
\text { McKimmy \& } \\
\text { Schmidt } \\
(2014,2015)\end{array}$ & $\begin{array}{l}\text { Hybrid virtual } \\
\text { classroom } \\
\text { Context: three } \\
\text { different cases at two } \\
\text { universities that }\end{array}$ & $\begin{array}{l}\text { Multiple case } \\
\text { study focusing on } \\
\text { design and } \\
\text { technical issues }\end{array}$ & $\begin{array}{l}\text { To illustrate different implementations } \\
\text { of Here or There instruction, explain the } \\
\text { affordances of these varied approaches, } \\
\text { provide best practices that are common } \\
\text { to each, and share lessons learned }\end{array}$ \\
\hline
\end{tabular}




\section{RQ 2: Benefits of synchronous hybrid learning}

Below we summarize the benefits that are indicated in previous state-of-the-art research. Based on textual data analysis, first, the research papers were explored inductively to generate categories of repeating benefits. The inductive process of identifying analytical categories as they emerge from the data is based on the grounded theory (Glaser \& Strauss, 1967). The data were read and reread to identify and index the found benefits. Through this process, benefits could be categorized into two categories, namely (1) organizational benefits related to educational access and efficiency in teaching; and (2) pedagogical benefits related to quality of learning.

\section{Organizational benefits}

Some higher educational institutions are dealing with a decline in student enrollment numbers due to the increased offering of distance and online education. The synchronous hybrid learning environment could provide an answer to this problem and help to increase the recruitment rates. This because, by offering the possibility to attend faceto-face or remote, the institution can reach out to a greater base of potential students (Abdelmalak \& Parra, 2016; Butz \& Askim-Lovseth, 2015; Ørngreen et al., 2015; Wang, Quek \& Hu, 2017; Wiles \& Ball, 2013) and, specifically for the hybrid virtual setting, it can ensure access to education regardless of place which provides more inclusive education and equality in learning outcomes (Bower, Dalgarno, Kennedy, Lee \& Kenney, 2015; Weitze, Ørngreen \& Levinsen, 2013). Moreover, it is possible to offer more elective or specific courses which are normally taught at one specific location and in addition external experts can be consulted more easily in the learning process, which can address the personal interests of the students and learners better (Bell, Sawaya \& Cain, 2014; McGovern \& Barnes, 2009). It can also ease workloads on a small educational team (Brumfield et al., 2017) as the hybrid virtual classroom eliminates the need to teach the same course twice to different classes at different campuses (Bell, Sawaya \& Cain, 2014; Wiles \& Ball, 2013). In addition, teachers and students do not have to move to the campus and consequently enjoy the freedom and flexibility this learning environment offers. Hence, one of the most cited benefits is flexibility in course attendance for the students. For example, when a student is ill or when he cannot move to the campus where the teacher will be present, there is the opportunity to follow remotely through online participation. This kind of flexibility is more in line with the current society we are living in (Lakhal, Bateman \& Bédard, 2017; Wang, Quek \& Hu, 2017; Wiles \& Ball, 2013). In addition, these learning environments accommodate for job \& family commitments and thus take a multifaceted student population in account (Lightner \& Lighnter-Laws, 2016; Wiley \& Ball, 2013).

\section{Pedagogical benefits}

The hybrid virtual classroom offers the possibility to include expertise outside the institution meaning that students are being exposed to a broader range of views and ideas, because this collaboration and connection between faceto-face and remote students creates richer learning experiences (Bell et al., 2014; Bower et al., 2015). Anastasiades et al. (2010) more specifically stressed that this setting can strengthen the social relations among students and teachers of the local and the remote class, and strengthen students' willingness to make new contacts all over the world. Also Liu, Spector, \& Ikle (2018) stressed that social benefit to students included providing equal learning opportunities to under-represented students. Likewise, this flexibility ensures that the synchronous hybrid learning environment can guarantee continuity of instruction and promotes student retention (Lakhal, Bateman \& Bédard, 2017; Ramsey, Evans \& Levy, 2016; Wang, Lang Quek \& Hu, 2017; Wiley \& Ball, 2013). Weitze, Ørngreen and Levinsen (2013) also mention this in their study:

For example the students own choice of environment helps them manage their family and everyday life by not always having to be present at school. Several students are also pleased with being able to vary their classroom environment during a day by changing geographical location, and when sitting at home they have the feeling that the school day ended sooner. The format also creates a new "intermediate solution" for some, when they feel "sluggish" and normally would have taken a sick-day. In this way, the concept contributes to their ability to complete their education. (Weitze, Ørngreen \& Levinsen, 2013, p. 5)

As mentioned in the citation, synchronous hybrid teaching offers the possibility to maintain the guidance and comfort of traditional courses for both remote students and for the students attending face-to-face. Moreover, by combining the two delivery modes, there is better support of the different learner characteristics and they can benefit from enhanced instruction and well-timed interactions (Szeto, 2014; Wiley \& Ball, 2013). Abdelmalak \& Parra (2016) moreover state that it give students a sense of control over their learning. 
Lastly, by teaching this way, students also encounter the many possibilities technology has to offer and they learn how to work with it. This can prepare them for careers in our technology-rich society (Butz \& Askim-Lovseth, 2015; Ørngreen et al., 2015).

Only limited studies have done empirical research to assess the differences between the outcomes of students who attend online versus in-person, yet the existing studies (Lightner \& Lightner-Laws, 2016; Szeto, 2014; White, Ramirez, Smith, \& Plonowski, 2010) provide evidence for the notion that flexible course delivery options have little to no negative impact on student learning as it results in similar learning outcomes, such as test scores (White et al. 2010), and motivation, needs satisfaction, and perceived success (Butz \& Stupnisky 2016)

\section{RQ3: Key challenges}

Apart from the above mentioned benefits, the synchronous hybrid learning also has many challenges.

This result section is divided into the two categories of challenges which are faced in the synchronous hybrid learning settings: i.e. pedagogical and technological challenges.

\section{Pedagogical challenges}

From the teacher perspective

This type of learning environment requires radical shifts in the teachers' pedagogical methods in order to accommodate to the new technology (Cain, 2015; Ramsey, Evans \& Levy, 2016). Weitze (2015) provided an adequate description of the influence technology has: "Although technologies are physical tools and not theoretical thinking tools or concepts, they change not only the way we carry out a task, but also the way we think about the task" (McLuhan, 1964; Hasse and Storgaard Brok, 2015 as found in Weitze, 2015, p. 1). The synchronous hybrid learning environments are a new kind of setup that highly influence the pedagogic and learning design (Weitze, Ørngreen \& Levinsen, 2013), and thus demand other methods of teaching and different activating learning activities (Bower et al., 2015). This means that the teacher or trainer has to adapt his teaching approach, but simultaneously has to maintain comparable learning standards (Grant \& Cheon, 2007; Lightner \& Lighnter-Laws, 2016). In addition, because the quality of the teaching is partly dependent on the teacher's or trainer's competence in using the technology (Bower et al., 2015), the teacher or trainer needs to actively learn how to work with the technology and has to get opportunities to try things out and evaluate the outcomes on the basis of evidence (Grant \& Cheon, 2007; Weitze et al., 2013).

Another challenge is that the synchronous hybrid learning environment requires more coordination from the teacher (Ørngreen et al., 2015). During the instruction in these new learning settings, the teacher needs to pay attention to both locations and also needs to perform certain operational actions on the teaching and learning platform. Hereby, the instructor has a heavy mental load, which is referred to as hyper-zoom or hyper-focus (Bower et al., 2015; Ørngreen et al., 2015; Zydney, McKimmy, Lindberg, \& Schmidt, 2019).

\section{From the student perspective}

When looking at the students' perspective in this new learning environment, research comparing the experiences of face-to-face students and remote students, found that these two groups experience the lesson differently in the hybrid synchronous situation (Beatty, 2007, 2010; Szeto, 2014; Zydney et al., 2019). Therefore, it is important to take this into account when preparing the learning experience. What drives the approach of synchronous hybrid learning is the desire to ensure all students are receiving comparable learning experiences regardless of location (Butz, Stupnisky, Pekrun, Jensen, \& Harsell, 2016). The challenges, however, lie in designing and implementing both pedagogical strategies and technological systems that enact those comparable learning experiences(Cain, Bell \& Cheng, 2016), also referred to as co-presence (Bower et al., 2014). For example, it is imperative that the teacher not only focuses his attention on the remote students and adopts a slower pace with lots of repetition, as these kind of strategies could compromise the class experience of the face-to-face students (Bower et al., 2015; Szeto, 2014).

The study of Olt (2018) specifically aimed to investigate the phenomenon of hybrid virtual learning from the perspective of the remote participant and concluded that the experience of the remote participant can be best explained and understood by the concept of 'ambiguity' in regard to group membership, functionality of technology, and place. Also Huang, Shu, Zhao, \& Huang (2017) showed that the remote students still felt excluded from the chief class, because they were physically separated from the F2F class, especially when the remote class encounters technical difficulties and without immediate support. Meanwhile, the F2F students felt neglected when teacher spent much time solving the technical problems. 
When implementing synchronous hybrid learning, it gets also more difficult to activate and engage the remote students to the same degree as the students attending face-to-face. In the study of Weitze (2015) both students and teachers state that remote students learned less, were generally more passive and often behaved like they were watching TV and not attending a lesson. One of the reasons for this finding is that teachers give classes based on more monologue-based teaching strategies, which are not well-suited for this kind of learning settings (Weitze, 2015).

In the study of Weitze et al. (2013) remote students indicated that it is difficult to make the teacher aware that they want to answer a question, which makes them frustrated and uninvolved. Therefore, it is important to take this into consideration in the design of the classes and to be aware that remote students need to be more invited into the class activity (Weitze et al., 2013). Further, remote learners feel a significant sense of distance from their institution. This illuminates the need to address the perceived distance between remote students and their teachers and face-to-face classmates by establishing some sort of connectedness (Ramsey, Evans \& Levy, 2016).

Lastly, the synchronous hybrid learning environment demands more self-discipline for students who are following remotely or online (Wiles \& Ball, 2013). Since the teacher is not physically present, there is less control of the students' engagement.

\section{Technological challenges}

An important question in relation to the pedagogical challenges is what the most effective technologies are for maximizing the social presence of remote students (Zydney et al., 2019). A disadvantage of the learning environment is the loss of visual and audible cues which normally are observable from the students (Weitze et al., 2013). Therefore, it is imperative that the teacher tries to ensure that the remote students always feel included in the class in order to reduce some of the distancing effects. For instance, the lecturer should frequently ask questions throughout the lesson and needs to be attentive to students' input (McGovern \& Barnes, 2009; Ørngreen et al., 2015).

The biggest challenge faced in the synchronous hybrid learning environment is the audio component which is decisive for the success (Bower et al., 2015; Cunningham, 2014; Zydney et al., 2019). Students who follow the class remotely or online should receive the same audio quality (and quality of education) as those students who are present face-to-face (McGovern \& Barnes, 2009). Therefore, setting up and testing the technology in advance is of great importance for the effectiveness of the class. A good idea is to let remote students log in prior to the session, so there is sufficient time to test and resolve possible problems (Bower et al., 2015).

In addition, the technology can be an imposition for the teacher and the F2F students, for instance if they need to be conscious of the orientation/positioning of cameras or are required to speak into a microphone interrupting conversational flow (Cunningham, 2014; Bower et al., 2015; Zydney et al., 2019). In these kind of innovative environments the technology is very visible, for example, the camera is visible and it is apparent when it is recording and streaming to remote students. This can make teachers very aware of their teaching performance and can cause them to act differently (Nortvig, 2013). In addition, these innovative technologies are continuously altered, which can be frustrating for teachers. It is found that small usability issues, caused by the continuous updates of innovative technologies, may confuse, delay or hinder the learning process of the students (Bell et al., 2014; Weitze, 2015).

Lastly, when students disappear from the screen because there is, for example a bad connection, this can induce the stress-level of the teacher. As a consequence, many teachers experience an immense fatigue after teaching in this learning settings (Weitze et al., 2013). Zydney et al. (2019) indicated that experienced instructors can facilitate both F2F and remote students without the aid of technical support when groups are limited to eight or fewer F2F students as a single omnidirectional speakerphone can adequately cover the area required to gather a class of this size around it and a swivl device can capture and display f2f students as they speak. However, larger class sizes will necessitate different approaches to facilitation.

\section{RQ4: Design guidelines}

\section{Design guidelines in response to challenges}

Training and support

Both the change in pedagogical methods and the use of technology ask for more preparation and organization, resulting in an increased workload (Bower et al., 2015; Wiles \& Ball, 2013). It is also important that the faculty 
provides sufficient training and support for academics, both pedagogically and technologically (Bower et al., 2015; Cain, 2015; Lightner \& Lightner-Laws, 2016; Szeto, 2014). As stated by Cain (2015), a possible solution for the many challenges the teacher faces, is the use of a technology navigator or operator. This person should be present in every class session to help troubleshoot problems both inside the classroom and online. The technology navigator or operator can also be consulted when the teacher had pedagogical questions. In the online platform, it should also be made possible for students to interact directly to the navigator or operator through, for example, a chat room (Cain, 2015; Cain, Bell \& Cheng, 2016). Also Zydney et al. (2019) suggest that students can take up more roles, such as "chat tracker" and "technology troubleshooter". They revealed that this solution not only take off some of the instructor's pressure to try to manage everything. Also, this can create a more student-centered learning environment, enabling more student ownership of the learning environment.

For students too, some kind of technological training on how to use and familiarize themselves with the online platform is required (McGovern \& Barnes, 2009; White et al., 2010). That way, they learn how to log on, enter a lesson and use all the tools the platform has to offer, such as silent questions or chat possibilities (Ramsey, Evans \& Levy, 2016). Further, adequate instructions must be provided to students. By communicating the need to purchase a headset, recommending students to connect through LAN rather than wirelessly, and asking them to run audio and video checks prior to the first lesson, the most typical problems can already be addressed outside of class (Ramsey, Evans \& Levy, 2016).

\section{Clear communication}

When a teacher decides to use the synchronous hybrid learning environment, Ørngreen et al. (2015) stress that a clear vision and expectations must be communicated to the students. For instance, it is a good idea to prepare alternative resolutions in advance and agree with students about what they should work on when a connection cannot be established (Grant \& Cheon, 2007). Next to communication about the technical requirements, a crucial pedagogical practice is to be explicit to students about how the hybrid synchronous sessions support the overall course learning objectives (Bower et al., 2014; Zydney et al., 2019).

It also is important to communicate very clearly what faculty staff can expect when teaching in a synchronous hybrid learning environment so they are prepared for the various challenges they will face and to make the different stakeholders collaborate (Weitze et al., 2013).

\section{Activating learners and curriculum alignment}

Possible solutions for the engagement problem are questioning of individuals through, for example, polls and presenting in an active and amusing manner (Bower et al., 2015). In addition, the lecturer should frequently ask questions throughout the lesson and needs to be attentive to students' input (McGovern \& Barnes, 2009; Ørngreen et al., 2015). Lastly, the significant sense of distance can be partly resolved by a virtual chat room or discussion forum. Through this, students are able to cooperate, share and contribute to each other's input (Ørngreen et al., 2015). In line with the clear communication about learning objectives and the fit with the overall curriculum, it is suggested that synchronous hybrid learning sessions are not organized as isolated sessions. As suggested by Zydney et al. (2019), hybrid synchronous sessions should built upon asynchronous activities (e.g. readings or performing exercises) from a flipped classroom approach. These authors also suggest to organize breakout sessions to create more student ownership of the learning environment. Bower et al. (2014) reported that copresence increases when the two cohorts of students are mixed during small group work or breakout sessions, however, they note this design may not always be desirable for practical reasons.

\section{RQ5: Gaps identified}

In line with several researchers (Abdelmalak \& Parra, 2016; Bower et al., 2015; Butz \& Askim-Lovseth, 2015; Butz \& Stupnisky, 2017; Olt, 2018; Zydney et al., 2019), this study can conclude that the research into synchronous hybrid learning is still in its infancy. As with any complex learning setting, initial development and research leads to many more questions. As an emerging practice, synchronous hybrid education especially needs increased empirical investigation to complement the qualitative case studies. Based on the results of this study the following directions for future work can be defined:

1. Future research including larger and more diverse samples to improve generalizability, but also provide additional statistical power to identify meaningful effects

2. Future research including more empirical and longitudinal data of the participants to investigate the impact of group membership over time. With multiple data points, future research could also endeavour to longitudinally predict students' assessment results based on learning activities. 
3. Future research including empirical realtime data of the learner experience as engagement, social presence or social belonging are multidimensional concepts difficult to measure. Next to self-report data, multimodal learning analytics could be used to better capture and compare students' experiences in different learning settings.

4. Future research including the effect on student learning and student outcomes across settings and specifically investigate the effectiveness of certain pedagogical scenarios (e.g. quizzes and polls, breakout sessions) for maximizing the learning experience and social presence of remote participants.

5. Future research investigating the most scalable approach with regard to technical and pedagogical capacity and limitations.

As stated by Bower et al., 2015 , ideally in the years to come, rich-media collaborative technologies will become so invisible that students and teachers interacting from different locations will feel as though they are in the same room. Yet, Liu et al. (2018) stress that we still have a long way to reach to these desired states.

\section{Conclusion}

This systematic review brought together the authors experiencing and investigating the benefits, challenges and guidelines regarding technological and pedagogical support for synchronous hybrid learning. We can conclude that existing research clearly shows the potential of this emerging practice. Despite the challenges, all studies express cautious optimism about synchronous hybrid or blended learning which creates a more flexible, engaging learning environment compared to fully online or fully on-site instruction. Based on our review, we can conclude that most of the existing literature is still exploratory and qualitative in nature and has focused mostly on the description of students' experiences, the organizational implementation and the technological design. Empirical studies have only begun to emerge and more research is needed examining different pedagogical scenarios and its impact on student outcomes.

\section{References}

Studies marked with $*$ are included in the literature review, see also Table 2

*Abdelmalak, M. M. M., \& Parra, J. L. (2016). Expanding Learning Opportunities for Graduate Students with HyFlex Course Design. International journal of online pedagogy and course design, 6(4), 19-37. https://doi.org/10.4018/IJOPCD.2016100102

*Alexander, M. M., Lynch, J. E., Rabinovich, T., \& Knutel, P. G. (2014). Snapshot of a hybrid learning environment. Quarterly Review of Distance Education, 15(1), 9-21.

*Anastasiades, P. S., Filippousis, G., Karvunis, L., Siakas, S., Tomazinakis, A., Giza, P., \& Mastoraki, H. (2010). Interactive Videoconferencing for Collaborative Learning at a Distance in the School of 21st Century: A Case Study in Elementary Schools in Greece. Computers \& Education, 54(2), 321-339.

Baker, C. K., \& Hjarlmarson, M. (2019). Designing Purposeful Student Interactions to Advance Synchronous Learning Experiences. International Journal of Web-Based Learning and Teaching Technologies, 14(1), 1-16.

Baumeister, R. F., \& Leary, M. R. (1997). Writing narrative literature reviews. Review of General Psychology, $1(3), 311$

*Beatty, B. J. (2007). Hybrid classes with flexible participation options - If you build it, how will they come? Paper presented at the 2007 Association for Educational Communications and Technology Annual Convention (October), Anaheim, CA.

*Beatty, B. (2010). Hybrid courses with flexible participation. Retrieved from http://itec.sfsu.edu/hyflex/ hyflex_course_design_theory_2.2.pdf

Bettany-Saltikov, J. (2010a). Learning how to undertake a systematic review: Part 1. Nursing Standard, 24(50), 47-55. 
Bettany-Saltikov, J. (2010b). Learning how to undertake a systematic review: Part 2. Nursing Standard, 24(51), $47-58$.

*Bell, J., Sawaya, S., \& Cain, W. (2014). Synchromodal classes: Designing for shared learning experiences between face-to-face and online students. International Journal of Designs for Learning, 5(1), 68-82.

Blau, G., Jarrell, S., McCloskey, M., Williams, W., Kerzner, A., \& Ford, T. (2018). Further Exploring Differences in Business Undergraduate Perceived Outcomes by Preferred Classroom Learning Environment. Journal of Education and Learning, 7(5), 20-30.

*Bower, M., Dalgarno, B., Kennedy, G. E., Lee, M. J., \& Kenney, J. (2014). Blended synchronous learning: A handbook for educators. Office for Learning and Teaching, Australian Department of Education.

*Bower, M., Dalgarno, B., Kennedy, G. E., Lee, M. J. W., \& Kenney, J. (2015). Design and implementation factors in blended synchronous learning environments: Outcomes from a cross-case analysis. Computers and Education, 86, 1-17. doi:10.1016/j.compedu.2015.03.006

*Bower, M., Lee, M. J., \& Dalgarno, B. (2017). Collaborative learning across physical and virtual worlds: Factors supporting and constraining learners in a blended reality environment. British Journal of Educational Technology, 48(2), 407-430. doi:10.1111/bjet.12435

*Butz, N. T., \& Askim-Lovseth, M. K. (2015). Oral communication skills assessment in a synchronous hybrid MBA programme: Does attending face-to-face matter for US and international students? Assessment and Evaluation in Higher Education, 40, 624-639. doi:10.1080/02602938.2014.940577

*Butz, N. T., Stupnisky, R. H., Pekrun, R., Jensen, J. L., \& Harsell, D. M. (2016). The Impact of emotions on student achievement in synchronous hybrid business and public administration programs: A longitudinal test of control-value theory. Decision Sciences Journal of Innovative Education, 14(4), 441-474.

*Butz, N. T., \& Stupnisky, R. H. (2016). A mixed methods study of graduate students' selfdetermined motivation in synchronous hybrid learning environments. The Internet and Higher Education, 28, 85-95.doi: 10.1016/j.iheduc.2015.10.003

*Butz, N. T., \& Stupnisky, R. H. (2017). Improving student relatedness through an online discussion intervention: The application of self-determination theory in synchronous hybrid programs. Computers and Education, 114, 117-138. doi:10.1016/j.compedu.2017.06.006

*Cain, W. (2015). Technology navigators: An innovative role in pedagogy, design and instructional support. In P. Redmond, J. Lock \& P. Danaher (Eds.), Educational innovations and contemporary technologies: Enhancing teaching and learning (pp.21-35). UK: Palgrave Macmillan.

*Cain, W., Bell, J., \& Cheng, C. (2016). Implementing robotic telepresence in a synchronous hybrid course. Proceedings - IEEE 16th International Conference on Advanced Learning Technologies, ICALT 2016, 171-175. doi:10.1109/ICALT.2016.79

*Cunningham, U. (2014). Teaching the disembodied: Othering and activity systems in a blended synchronous learning situation. International Review of Research in Open and Distributed Learning, 15(6), 33-51.

Glaser, B.G. \& Strauss, A.L. (1967). The discovery of grounded theory: Strategies for Qualitative research. Chicago, IL: Aldine Publishing. doi:10.1097/00006199-196807000- 00014

*Grant, M. M., \& Cheon, J. (2007). The value of using synchronous conferencing for instruction and students. Journal of Interactive Online Learning, 6(3), 211-226.

*Hastie, M., Hung, I. C., Chen, N. S., \& Kinshuk. (2010). A blended synchronous learning model for educational international collaboration. Innovations in Education and Teaching International, 47(1), 9-24. 
doi:10.1080/14703290903525812

*Huang, Y., Shu, F., Zhao, C., \& Huang, J. (2017). Investigating and Analyzing Teaching Effect of Blended Synchronous Classroom. In Liu, J and Nishimura, S and Zhang, H and Jin, Q (Ed.), 2017 6TH International Conference of Educational Innovation Through Technology (EITT) (pp. 134-135). https://doi.org/10.1109/EITT.2017.40

[Name omitted for blind review]. (2018). Strategic plan in 5 projects: Going digital.

*Lakhal, S., Bateman, D., \& Bédard, J. (2017). Blended synchronous delivery modes in graduate programs: A literature review and its implementation in the master teacher program. Collected Essays on Learning and Teaching, 10, 47-60. doi:10.22329/celt.v10i0.4747

*Lightner, C. A., \& Lightner-Laws, C. A. (2016). A blended model: Simultaneously teaching a quantitative course traditionally, online, and remotely. Interactive Learning Environments, 24, 224-238. doi:10.1080/10494820.2013.841262

*Liu, H., Spector, J. M., \& Ikle, M. (2018). Computer technologies for model-based collaborative learning: A research-based approach with initial findings. Computer Applications in Engineering Education, 26(5, SI), 1383-1392. https://doi.org/10.1002/cae.22049

*McGovern, N., \& Barnes, K. (2009). Lectures from my living room: A pilot study of hybrid learning from the students' perspective. In F. L Wang, J. Fong, L. Zhang \& V. S.K. Lee (Eds.), Hybrid learning and education (pp. 284-298). Berlin, Germany: Springer.

*McKimmy, P. B., \& Schmidt, M. (2014) HOT Classroom: Iterations on equipping a here-or-there instructional space. Presented at the 2014 International Convention of the Association for Educational Communications and Technology. Jacksonville, FL.

*McKimmy, P.B., \& Schmidt, M. (2015). HOT instruction: Equipping a here-or-there classroom. Presented at the 20th Annual Technology, Colleges \& Community Worldwide Conference. Honolulu, HI.

Moher D, Liberati A, Tetzlaff J, Altman DG, \& The PRISMA Group. (2009). Preferred reporting items for systematic reviews and meta-analyses: The PRISMA statement. PLoS Med 6(7): e1000097. doi:10.1371/journal.pmed.1000097

*Nortvig, A.-M. (2013). In the presence of technology - teaching in hybrid synchronous classrooms. Proceedings of the European Conference on E-Learning, ECEL, 347-353.

*Olt, P. A. (2018). Virtually There: Distant Freshmen Blended in Classes through Synchronous Online Education. Innovative Higher Education, 43(5), 381-395. https://doi.org/10.1007/s10755-018-9437-z

* Ørngreen, R., Levinsen, K., Jelsbak, V., Moller, K. L., \& Bendsen, T. (2015). Simultaneous class-based and live video streamed teaching: Experiences and derived principles from the bachelor programme in biomedical laboratory analysis. In A. Jefferies \& M. Cubric (Eds.), Proceedings of the $14^{\text {th }}$ european conference on E-learning (ECEL 2015) (pp. 451-459). UK: Academic Conferences and Publishing International Limited.*Ramsey, D., Evans, J., \& Levy, M. (2016). Preserving the Seminar Experience. Journal of Political Science Education, 12(3), 256-267. doi:10.1080/15512169.2015.1077713

*Rasmussen, R. C. (2003). The quantity and quality of human interaction in a synchronous blended learning environment. (Doctoral dissertation, Brigham Young University) Available from ProQuest Dissertations $\&$ theses. (UMI No. 305345928)

*Romero-Hall, E., \& Vicentini, C. (2017). Examining Distance Learners in Hybrid Synchronous Instruction: Successes and Challenges. ONLINE LEARNING, 21(4, SI), 141-157.

*Roseth, C., Akcaoglu, M., \& Zellner, A. (2013). Blending synchronous face-to-face and computer-supported 
cooperative learning in a hybrid doctoral seminar. TechTrends, 57(3), 54-59. doi:10.1007/s11528-013-0663-z

*Shen, R. M., Wang, M. J., \& Pan, X. (2008). Increasing interactivity in large blended classrooms through a cutting-edge mobile learning system. British Journal of Educational Technology, 39(6), 1073-1086. https://doi.org/10.1109/ITICT.2008.4806642

*Stewart, A. R., Harlow, D. B., \& DeBacco, K. (2011). Students' experience of synchronous learning in distributed environments. Distance $\quad$ Education, 32(3), 357-381. https://doi.org/10.1080/01587919.2011.610289

*Szeto, E. (2014). A Comparison of online/face-to-face students' and instructor's experiences: Examining blended synchronous learning effects. Procedia - Social and Behavioral Sciences, 116, 4250-4254. doi:10.1016/j.sbspro.2014.01.926

*Szeto, E. (2015). Community of inquiry as an instructional approach: What effects of teaching, social and cognitive presences are there in blended synchronous learning and teaching? Computers and Education, 81, 191-201. doi:10.1016/j.compedu.2014.10.015

*Szeto, E., \& Cheng, A. Y. N. (2016). Towards a framework of interactions in a blended synchronous learning environment: What effects are there on students' social presence experience? Interactive Learning Environments, 24(3), 487-503. doi:10.1080/10494820.2014.881391

*Vu, P., \& Fadde, P. J. (2013). When to Talk, When to Chat: Student Interactions in Live Virtual Classrooms. Journal of Interactive Online Learning, 12(2). Retrieved from www.ncolr.org/jio

*Wang, Q., \& Huang, C. (2018). Pedagogical, social and technical designs of a blended synchronous learning environment. British Journal of Educational Technology, 49(3), 451-462. doi:10.1111/bjet.12558

*Wang, Q., Huang, C., \& Quek, C. L. (2018). Students' perspectives on the design and implementation of a blended synchronous learning environment. Australasian Journal of Educational Technology, 34(1), 113. doi: $10.14742 /$ ajet.3404

*Wang, Q., Quek, C. L., \& Hu, X. (2017). Designing and improving a blended synchronous learning environment: An educational design research. International Review of Research in Open and Distributed Learning, 18(3), 99-118.

*Weitze, C. L. (2015). Pedagogical innovation in teacher teams: An organisational learning design model for continuous competence development. In I A. Jefferies, \& M. Cubric (Eds.), Proceedings of 14th European Conference on e-Learning ECEL-2015 (s. 629-638). Reading, UK: Academic Conferences and Publishing International. Proceedings of the European Conference on e-Learning.

*Weitze, C. L., Ørngreen, R., \& Levinsen, K. (2013). The global classroom video conferencing model and first evaluations. In I M. Ciussi, \& M. Augier (Eds.), Proceedings of the 12th European Conference on ELearning: SKEMA Business School, Sophia Antipolis France, 30-31 October 2013 (Bind 2, s. 503-510). Reading, UK: Academic Conferences and Publishing International.

*White, C. P., Ramirez, R., Smith, J. G., \& Plonowski, L. (2010). Simultaneous delivery of a face-to-face course to on-campus and remote off-campus students. TechTrends, 54(4), 34-40. doi: 10.1007/s11528-0100418-z

*Wiles, G. L., \& Ball, T. R. (2013, June 23-26). The converged classroom. Paper presented at 2013 ASEE Annual Conference: Improving course effectiveness, Atlanta, Georgia. https://peer.asee.org/22561

*Yen, C.-J., \& Abdous, M. (2012). A Study of the predictive relationships between faculty engagement, learner satisfaction and outcomes in multiple learning delivery modes. International Journal of Distance Education Technologies, 10(1), 74-87. doi:10.4018/jdet.2011100105 
*Zydney, J. M., McKimm, P., Lindberg, R., \& Schmidt, M. (2019). Here or There Instruction: Lessons Learned in Implementing Innovative Approaches to Blended Synchronous Learning. TECHTRENDS, 63(2), 123132. https://doi.org/10.1007/s11528-018-0344-z 